\title{
Thermochemistry and Kinetics of Hydrogen Abstraction by Methyl Radical from Polycyclic Aromatic Hydrocarbons
}

\author{
Karen Hemelsoet, ${ }^{* \dagger,+}$ Veronique Van Speybroeck, ${ }^{\dagger}$ Damian Moran, ${ }^{\ddagger, \mathbb{I}}$ Guy B. Marin, ${ }^{\S}$ \\ Leo Radom, ${ }^{*,+, \text { Il }}$ and Michel Waroquier ${ }^{*, \dagger}$ \\ Center for Molecular Modeling, Ghent University, Proeftuinstraat 86, B-9000 Ghent, Belgium, \\ School of Chemistry and ARC Centre of Excellence in Free Radical Chemistry and Biotechnology, \\ University of Sydney, Sydney, NSW 2006, Australia, and Laboratorium voor Petrochemische \\ Techniek, Ghent University, Krijgslaan 281-S5, B-9000 Ghent, Belgium
}

\section{SUPPORTING INFORMATION}

(Figures S1-S2, Tables S1-S3, Total 16 pages)

\footnotetext{
*To whom correspondence should be addressed. E-mail:

karen.hemelsoet@UGent.be, radom@chem.usyd.edu.au, michel.waroquier@UGent.be

${ }^{\dagger}$ Center for Molecular Modeling

University of Sydney

"ARC Centre of Excellence in Free Radical Chemistry and Biotechnology

${ }^{\S}$ Laboratorium voor Petrochemische Techniek
} 
Figure S1: Reaction enthalpies $\left(\Delta H_{298}\right)$ for hydrogen abstraction by methyl radical from possible sites of PAHs (BMK/6-311+G(3df,2p)//B3-LYP/6-311G(d,p), $\left.\mathrm{kJ} \mathrm{mol}^{-1}\right)$.

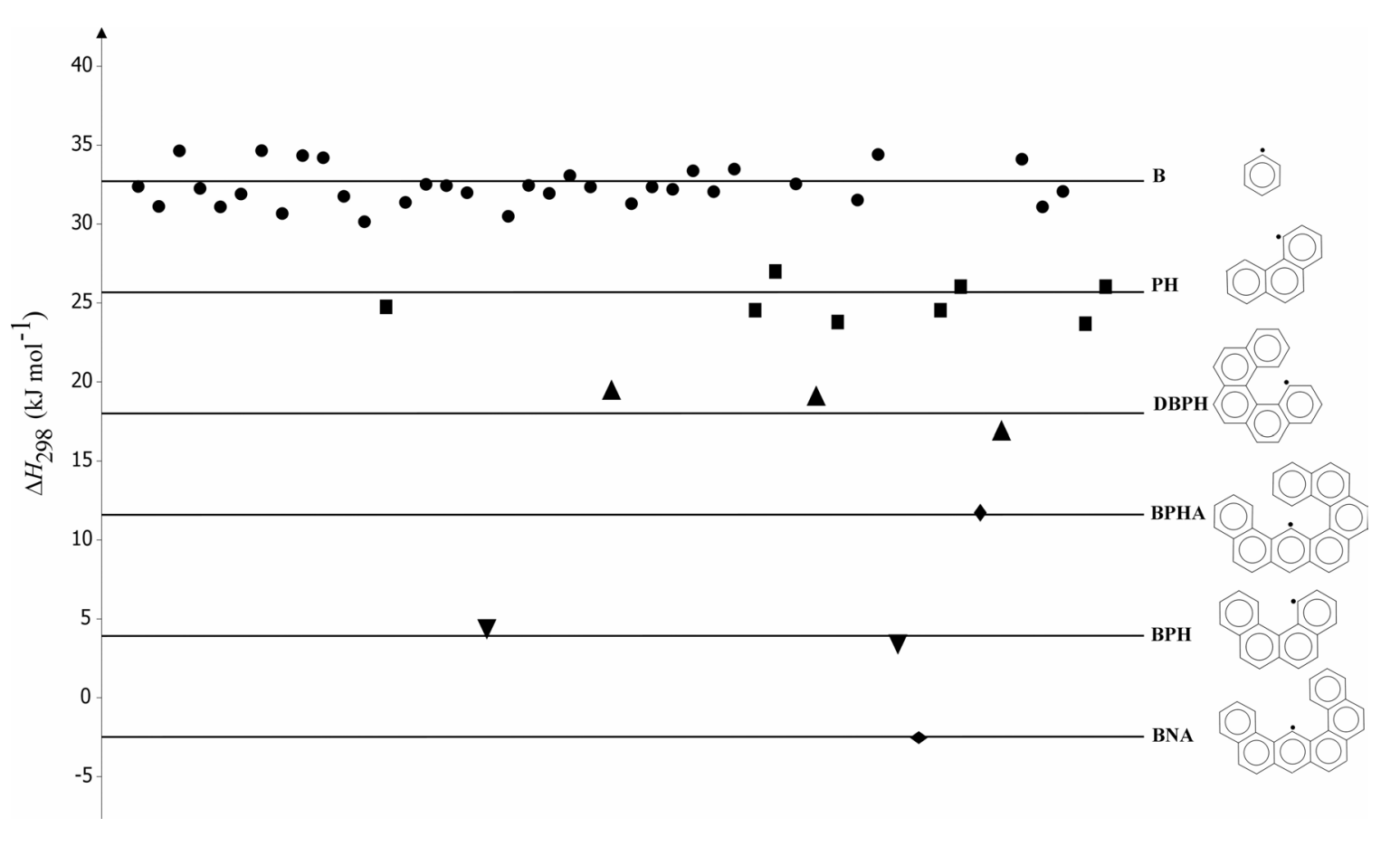




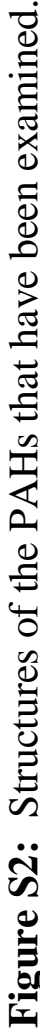

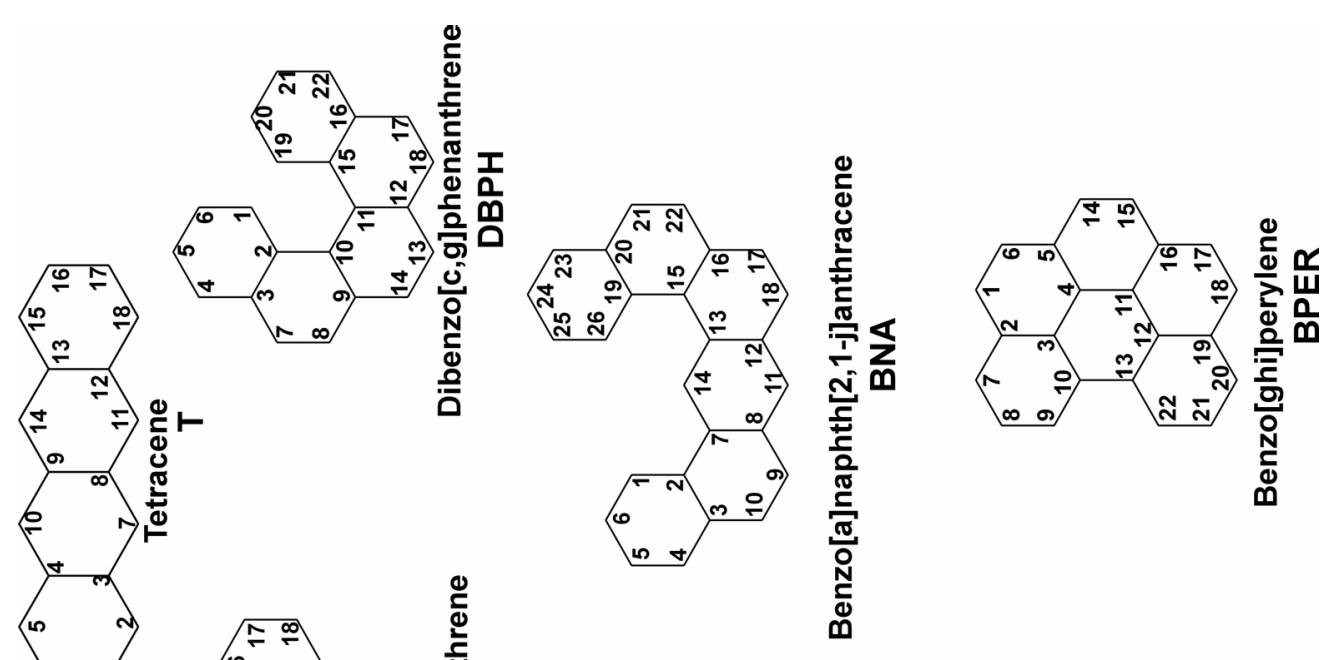

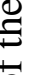

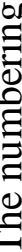

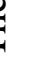
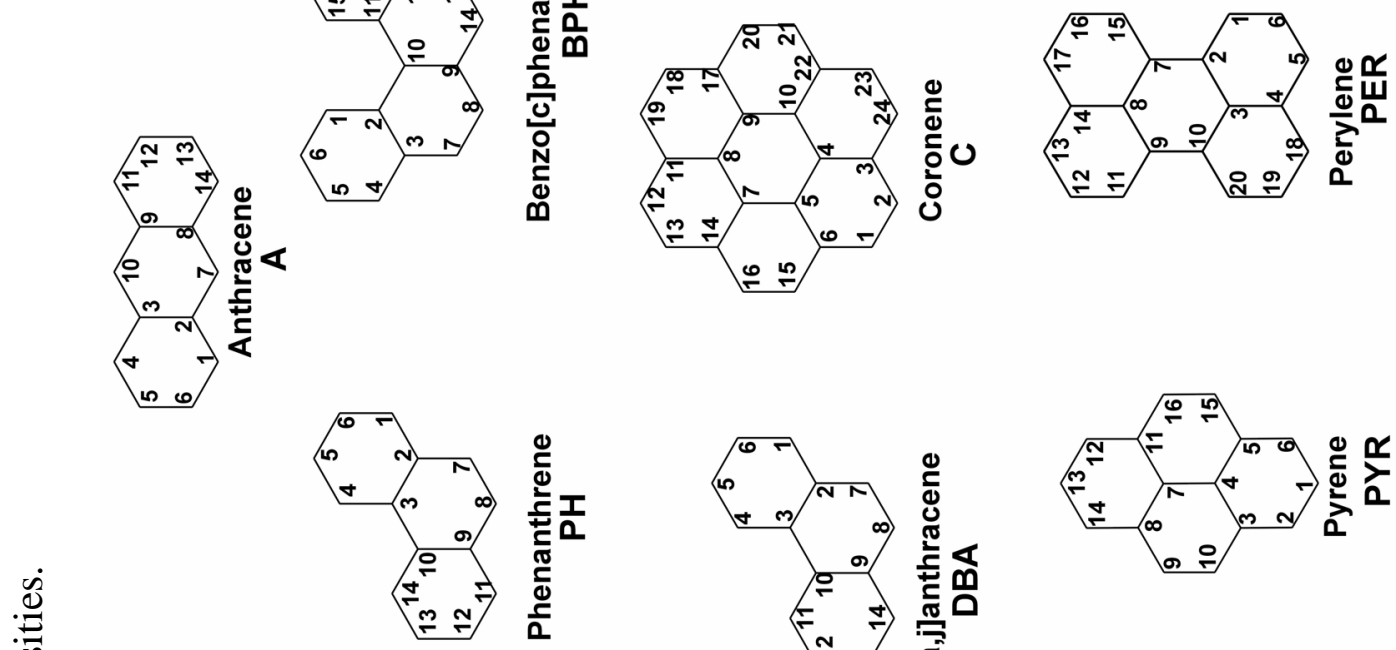

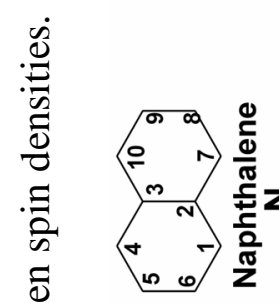
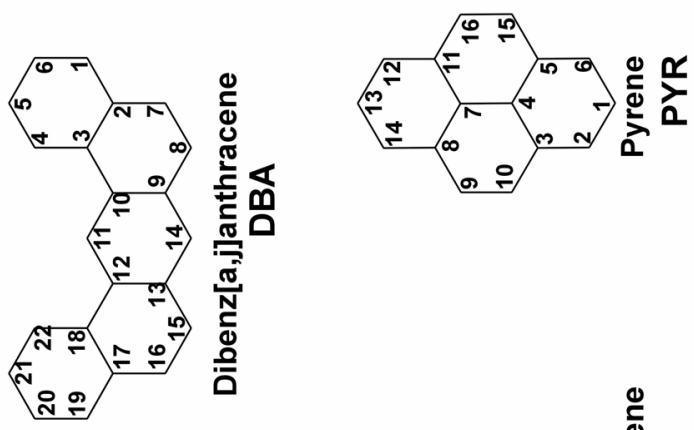

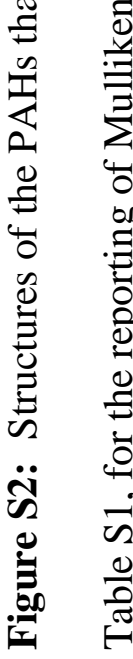
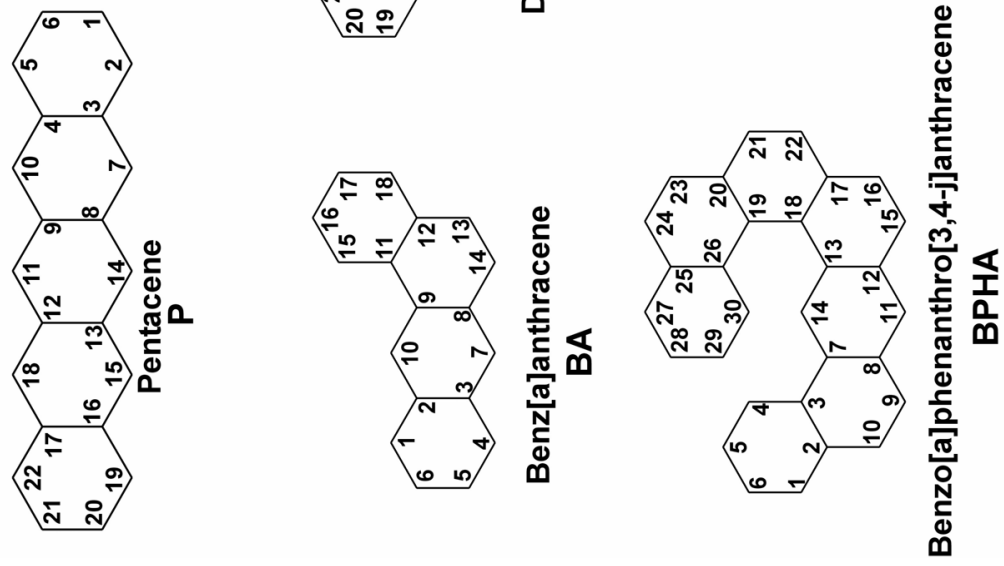
TABLE S1: Mulliken Spin Densities, in Condensed-to-Atoms Form, for the Polycyclic Aryl Radicals (BMK/6-311+G(3df,2p)//B3-LYP/6-311G(d,p) ${ }^{a}$

\begin{tabular}{|c|c|c|c|c|c|c|c|c|c|}
\hline & B-1 & $\mathbf{N}-1$ & $\mathbf{N}-2$ & A-1 & A-2 & A-3 & T-1 & $\mathbf{T}-2$ & T-3 \\
\hline 1 & 0.059 & -0.009 & -0.081 & -0.018 & -0.010 & 0.011 & 0.023 & 0.011 & -0.012 \\
\hline 2 & -0.061 & 0.059 & 0.062 & 0.071 & 0.012 & -0.015 & -0.022 & -0.010 & 0.011 \\
\hline 3 & 0.983 & -0.058 & -0.028 & -0.073 & -0.016 & 0.013 & 0.026 & 0.012 & -0.014 \\
\hline 4 & -0.061 & 0.029 & 0.058 & 0.041 & 0.011 & -0.010 & -0.033 & -0.013 & 0.012 \\
\hline 5 & 0.059 & -0.018 & -0.049 & -0.030 & -0.011 & 0.009 & 0.024 & 0.011 & -0.011 \\
\hline 6 & -0.042 & 0.013 & 0.988 & 0.021 & 0.010 & -0.013 & -0.025 & -0.011 & 0.010 \\
\hline 7 & & -0.049 & -0.011 & -0.068 & -0.020 & 0.021 & -0.046 & -0.023 & 0.023 \\
\hline 8 & & 0.068 & 0.013 & 0.071 & 0.061 & -0.026 & 0.078 & 0.015 & -0.017 \\
\hline 9 & & -0.077 & -0.019 & -0.073 & -0.057 & 0.067 & -0.075 & -0.019 & 0.017 \\
\hline 10 & & 0.994 & 0.014 & 1.014 & 0.038 & -0.024 & 0.068 & 0.024 & -0.024 \\
\hline 11 & & & & -0.018 & 0.999 & -0.093 & -0.092 & -0.032 & 0.033 \\
\hline 12 & & & & 0.021 & -0.088 & 0.995 & 0.085 & 0.067 & -0.029 \\
\hline 13 & & & & -0.030 & 0.076 & -0.047 & -0.090 & -0.061 & 0.074 \\
\hline 14 & & & & 0.041 & -0.055 & 0.060 & 1.035 & 0.051 & -0.038 \\
\hline 15 & & & & & & & 0.054 & 1.005 & -0.102 \\
\hline 16 & & & & & & & -0.044 & -0.098 & 1.001 \\
\hline 17 & & & & & & & 0.033 & 0.083 & -0.051 \\
\hline 18 & & & & & & & -0.030 & -0.061 & 0.065 \\
\hline
\end{tabular}


TABLE S1: continued ...

\begin{tabular}{|c|c|c|c|c|c|c|c|c|c|}
\hline & P-1 & P-2 & P-3 & P-4 & PH-1 & PH-2 & PH-3 & PH-4 & PH-5 \\
\hline 1 & 0.051 & 0.056 & 0.096 & -0.062 & -0.045 & 0.058 & -0.074 & 0.993 & 0.024 \\
\hline 2 & -0.049 & -0.051 & -0.073 & 0.076 & 0.061 & -0.033 & 0.061 & -0.065 & -0.050 \\
\hline 3 & 0.058 & 0.111 & 0.079 & -0.041 & -0.068 & 0.063 & -0.035 & 0.063 & 0.055 \\
\hline 4 & -0.066 & -0.116 & -0.073 & 0.088 & 0.985 & -0.081 & 0.059 & -0.046 & -0.005 \\
\hline 5 & 0.051 & 0.077 & 1.015 & -0.115 & -0.072 & 0.991 & -0.051 & 0.065 & 0.011 \\
\hline 6 & -0.054 & -0.067 & -0.111 & 1.012 & 0.066 & -0.050 & 0.986 & -0.070 & -0.012 \\
\hline 7 & -0.103 & -0.136 & -0.057 & 0.056 & -0.003 & 0.013 & -0.004 & 0.028 & 0.994 \\
\hline 8 & 0.111 & 0.101 & 0.027 & -0.029 & 0.010 & -0.019 & 0.011 & -0.020 & -0.090 \\
\hline 9 & -0.109 & -0.096 & -0.031 & 0.030 & -0.010 & 0.008 & -0.009 & 0.008 & 0.067 \\
\hline 10 & 0.126 & 1.074 & 0.075 & -0.063 & 0.030 & -0.003 & 0.010 & -0.002 & -0.028 \\
\hline 11 & 1.102 & 0.128 & 0.055 & -0.057 & 0.005 & -0.004 & 0.006 & -0.005 & -0.012 \\
\hline 12 & -0.109 & -0.052 & -0.024 & 0.024 & -0.007 & 0.005 & -0.008 & 0.005 & 0.012 \\
\hline 13 & 0.111 & 0.047 & 0.023 & -0.025 & 0.003 & -0.005 & 0.005 & -0.005 & -0.019 \\
\hline 14 & -0.168 & -0.107 & -0.054 & 0.054 & 0.000 & 0.006 & -0.007 & 0.007 & 0.013 \\
\hline 15 & -0.103 & -0.076 & -0.037 & 0.038 & & & & & \\
\hline 16 & 0.058 & 0.042 & 0.021 & -0.022 & & & & & \\
\hline 17 & -0.066 & -0.044 & -0.021 & 0.021 & & & & & \\
\hline 18 & 0.126 & 0.078 & 0.038 & -0.039 & & & & & \\
\hline 19 & -0.049 & -0.035 & -0.017 & 0.018 & & & & & \\
\hline 20 & 0.051 & 0.037 & 0.018 & -0.019 & & & & & \\
\hline 21 & -0.054 & -0.038 & -0.018 & 0.018 & & & & & \\
\hline
\end{tabular}


TABLE S1: continued ...

$\begin{array}{lllll}22 & 0.051 & 0.036 & 0.018 & -0.018\end{array}$

\begin{tabular}{|c|c|c|c|c|c|c|}
\hline & ВPH-1 & ВРН-2 & ВРH-3 & ВРН-4 & ВРН-5 & ВРН-6 \\
\hline 1 & 0.047 & -0.004 & 0.003 & -0.003 & -0.008 & 0.006 \\
\hline 2 & -0.005 & 0.004 & -0.004 & 0.005 & 0.010 & -0.001 \\
\hline 3 & 0.006 & -0.004 & 0.003 & -0.003 & -0.010 & 0.007 \\
\hline 4 & -0.006 & 0.003 & -0.002 & 0.002 & 0.007 & -0.005 \\
\hline 5 & 0.007 & -0.004 & 0.002 & -0.003 & -0.009 & 0.005 \\
\hline 6 & -0.005 & 0.004 & -0.002 & 0.002 & 0.006 & -0.004 \\
\hline 7 & -0.009 & 0.006 & -0.009 & 0.005 & 0.012 & -0.013 \\
\hline 8 & 0.006 & -0.004 & 0.006 & -0.004 & -0.006 & 0.024 \\
\hline 9 & -0.013 & 0.011 & -0.012 & 0.010 & 0.069 & -0.055 \\
\hline 10 & 0.031 & -0.009 & 0.012 & -0.006 & -0.037 & 0.060 \\
\hline 11 & -0.069 & 0.066 & -0.033 & 0.064 & 0.057 & -0.028 \\
\hline 12 & 0.060 & -0.033 & 0.062 & -0.065 & -0.051 & 0.068 \\
\hline 13 & -0.006 & 0.015 & -0.006 & 0.029 & 0.991 & -0.085 \\
\hline 14 & 0.011 & -0.020 & 0.012 & -0.020 & -0.085 & 0.991 \\
\hline 15 & 0.965 & -0.082 & 0.059 & -0.047 & -0.007 & 0.014 \\
\hline 16 & -0.076 & 0.987 & -0.050 & 0.068 & 0.012 & -0.019 \\
\hline 17 & 0.067 & -0.051 & 0.985 & -0.071 & -0.013 & 0.013 \\
\hline 18 & -0.047 & 0.062 & -0.075 & 0.991 & 0.026 & -0.012 \\
\hline
\end{tabular}


TABLE S1: continued ...

\begin{tabular}{|c|c|c|c|c|c|c|c|c|c|}
\hline & DBPH-1 & DBPH-2 & DBPH-3 & DBPH-4 & DBPH-5 & DBPH-6 & DBPH-7 & DBA-1 & DBA-2 \\
\hline 1 & -0.029 & 0.002 & -0.002 & 0.001 & 0.003 & -0.002 & -0.008 & 0.007 & -0.001 \\
\hline 2 & 0.025 & -0.003 & 0.002 & -0.003 & -0.005 & 0.005 & 0.011 & -0.012 & 0.002 \\
\hline 3 & -0.013 & 0.002 & -0.002 & 0.002 & 0.004 & -0.002 & -0.011 & 0.036 & -0.002 \\
\hline 4 & 0.036 & -0.001 & 0.001 & -0.001 & -0.003 & 0.002 & 0.007 & -0.003 & 0.002 \\
\hline 5 & -0.017 & 0.002 & -0.001 & 0.001 & 0.003 & -0.002 & -0.009 & 0.005 & -0.001 \\
\hline 6 & 0.041 & -0.001 & 0.001 & -0.001 & -0.003 & 0.002 & 0.006 & -0.009 & 0.001 \\
\hline 7 & 0.007 & -0.003 & 0.002 & -0.003 & -0.010 & 0.005 & 0.013 & 0.012 & -0.004 \\
\hline 8 & -0.008 & 0.002 & -0.002 & 0.002 & 0.006 & -0.004 & -0.006 & -0.005 & 0.003 \\
\hline 9 & 0.009 & -0.004 & 0.004 & -0.003 & -0.013 & 0.009 & 0.070 & 0.066 & -0.006 \\
\hline 10 & -0.014 & 0.005 & -0.006 & 0.006 & 0.012 & -0.004 & -0.038 & -0.081 & 0.001 \\
\hline 11 & 0.032 & -0.008 & 0.012 & -0.005 & -0.035 & 0.064 & 0.061 & 1.001 & -0.001 \\
\hline 12 & -0.014 & 0.010 & -0.012 & 0.009 & 0.068 & -0.057 & -0.055 & -0.081 & 0.033 \\
\hline 13 & 0.009 & -0.004 & 0.006 & -0.005 & -0.008 & 0.025 & 0.990 & 0.066 & -0.009 \\
\hline 14 & -0.011 & 0.006 & -0.009 & 0.005 & 0.013 & -0.014 & -0.081 & -0.050 & 0.006 \\
\hline 15 & -0.069 & 0.066 & -0.034 & 0.064 & 0.056 & -0.030 & -0.002 & -0.005 & 0.010 \\
\hline 16 & 0.057 & -0.032 & 0.062 & -0.065 & -0.050 & 0.071 & 0.007 & 0.012 & -0.002 \\
\hline 17 & -0.006 & 0.014 & -0.006 & 0.029 & 0.992 & -0.087 & -0.014 & -0.012 & 0.062 \\
\hline 18 & 0.012 & -0.019 & 0.012 & -0.019 & -0.086 & 0.989 & 0.024 & 0.036 & -0.073 \\
\hline 19 & 0.961 & -0.082 & 0.059 & -0.047 & -0.006 & 0.014 & 0.007 & 0.007 & -0.044 \\
\hline 20 & -0.072 & 0.987 & -0.050 & 0.069 & 0.012 & -0.020 & -0.004 & -0.009 & 0.066 \\
\hline
\end{tabular}


TABLE S1: continued ...

$$
\begin{array}{rrrrrrrrrr}
21 & 0.064 & -0.051 & \mathbf{0 . 9 8 5} & -0.072 & -0.013 & 0.015 & 0.005 & 0.005 & -0.070 \\
22 & -0.044 & 0.060 & -0.075 & \mathbf{0 . 9 9 2} & 0.025 & -0.012 & -0.005 & -0.003 & \mathbf{0 . 9 8 5}
\end{array}
$$

\begin{tabular}{lccccccccc}
\hline & DBA-3 & PYR-1 & PYR-2 & PYR-3 & PER-1 & BPER-1 & BA-1 & BA-2 & BA-3 \\
\hline 1 & 0.059 & 0.010 & -0.009 & 0.014 & -0.017 & 0.016 & -0.004 & 0.036 & $\mathbf{0 . 9 9 3}$ \\
2 & -0.034 & -0.018 & 0.009 & -0.018 & 0.014 & -0.036 & 0.001 & -0.069 & -0.054 \\
3 & 0.064 & 0.012 & -0.009 & 0.069 & -0.012 & 0.071 & -0.005 & 0.065 & 0.061 \\
4 & -0.081 & -0.003 & 0.008 & -0.025 & 0.009 & -0.004 & 0.004 & -0.013 & -0.052 \\
5 & $\mathbf{0 . 9 9 2}$ & 0.012 & -0.009 & 0.012 & -0.014 & 0.011 & -0.005 & 0.017 & 0.072 \\
6 & -0.052 & -0.017 & 0.009 & -0.023 & 0.009 & -0.021 & 0.003 & -0.024 & -0.083 \\
7 & 0.014 & 0.066 & -0.028 & 0.055 & -0.015 & 0.068 & 0.007 & -0.057 & -0.015 \\
8 & -0.020 & -0.072 & 0.059 & -0.051 & 0.072 & -0.060 & -0.008 & 0.072 & 0.012 \\
9 & 0.007 & 0.033 & -0.004 & $\mathbf{0 . 9 9 6}$ & -0.100 & $\mathbf{0 . 9 9 4}$ & 0.032 & -0.083 & -0.019 \\
10 & -0.003 & -0.025 & 0.011 & -0.093 & 0.045 & -0.088 & -0.004 & $\mathbf{1 . 0 0 3}$ & 0.033 \\
11 & 0.007 & -0.039 & 0.059 & -0.004 & $\mathbf{1 . 0 0 0}$ & 0.009 & -0.072 & 0.037 & 0.007 \\
12 & -0.005 & 0.069 & -0.065 & 0.014 & -0.056 & -0.010 & 0.062 & -0.014 & -0.005 \\
13 & 0.004 & -0.063 & $\mathbf{0 . 9 8 5}$ & -0.012 & 0.071 & 0.037 & -0.002 & 0.015 & 0.008 \\
14 & -0.006 & $\mathbf{1 . 0 0 0}$ & -0.065 & 0.028 & -0.032 & -0.008 & 0.010 & -0.007 & -0.006 \\
15 & -0.002 & -0.023 & 0.011 & -0.008 & 0.020 & 0.007 & $\mathbf{0 . 9 8 5}$ & -0.004 & -0.005 \\
16 & 0.003 & 0.018 & -0.004 & 0.010 & -0.020 & -0.009 & -0.069 & 0.006 & 0.003 \\
& -0.002 & & & & 0.021 & 0.006 & 0.065 & -0.011 & -0.004
\end{tabular}


TABLE S1： continued ...

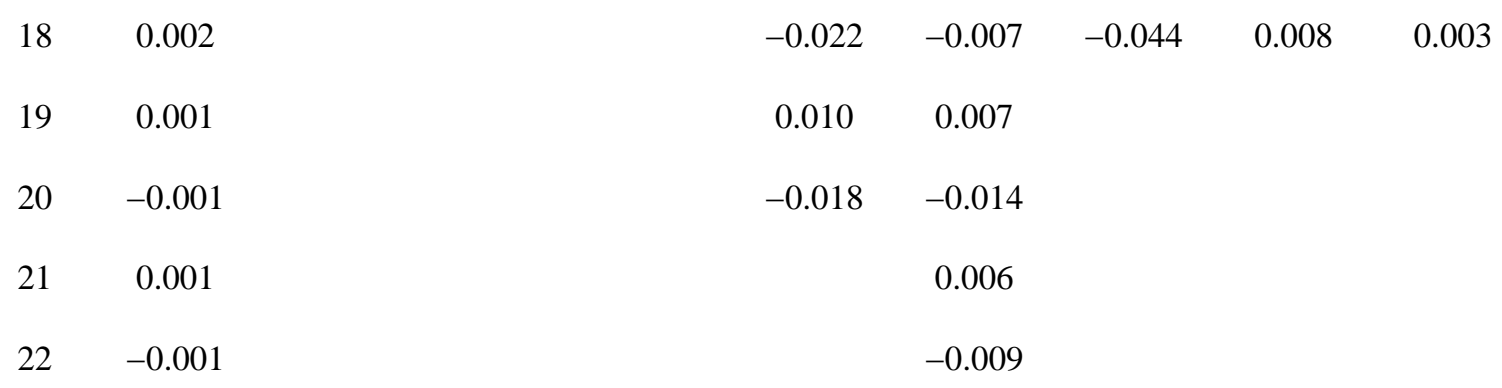

\begin{tabular}{lccccccc}
\hline & C-1 & BNA-1 & BNA-2 & BNA-3 & BPHA-1 & BPHA-2 & BPHA-3 \\
\hline 1 & 0.005 & 0.000 & -0.007 & $\mathbf{0 . 9 8 5}$ & -0.044 & 0.007 & -0.006 \\
2 & -0.005 & 0.003 & 0.038 & -0.072 & 0.062 & -0.012 & 0.010 \\
3 & 0.006 & -0.002 & -0.013 & 0.062 & -0.072 & 0.035 & -0.012 \\
4 & -0.006 & 0.001 & 0.007 & -0.044 & $\mathbf{0 . 9 8 6}$ & -0.005 & 0.009 \\
5 & 0.006 & -0.001 & -0.010 & 0.065 & -0.070 & 0.006 & -0.005 \\
6 & -0.005 & 0.001 & 0.006 & -0.069 & 0.065 & -0.010 & 0.009 \\
7 & -0.002 & -0.006 & -0.090 & 0.032 & 0.032 & -0.079 & 0.044 \\
8 & 0.062 & 0.006 & 0.068 & -0.009 & -0.009 & 0.067 & -0.019 \\
9 & -0.029 & -0.001 & -0.006 & 0.010 & 0.010 & -0.006 & 0.009 \\
10 & 0.010 & 0.002 & 0.013 & -0.002 & -0.002 & 0.014 & -0.010 \\
11 & -0.059 & -0.007 & -0.053 & 0.006 & 0.006 & -0.050 & 0.053 \\
12 & 0.026 & 0.007 & 0.067 & -0.005 & -0.006 & 0.063 & -0.022 \\
13 & -0.015 & -0.007 & -0.078 & 0.002 & 0.002 & -0.080 & 0.040 \\
14 & 0.009 & 0.056 & $\mathbf{0 . 9 9 0}$ & -0.002 & -0.003 & $\mathbf{0 . 9 8 3}$ & -0.044
\end{tabular}


TABLE S1: continued ...

\begin{tabular}{|c|c|c|c|c|c|c|c|}
\hline 15 & 0.006 & 0.034 & 0.037 & -0.003 & 0.003 & -0.008 & 0.012 \\
\hline 16 & -0.007 & -0.014 & -0.016 & 0.002 & -0.004 & 0.014 & -0.013 \\
\hline 17 & 0.065 & 0.007 & 0.014 & -0.004 & 0.002 & -0.016 & 0.014 \\
\hline 18 & -0.079 & -0.009 & -0.008 & 0.003 & -0.003 & 0.038 & -0.020 \\
\hline 19 & 0.993 & -0.070 & -0.007 & 0.001 & 0.001 & -0.014 & 0.035 \\
\hline 20 & -0.008 & 0.060 & 0.008 & -0.001 & -0.001 & 0.008 & -0.015 \\
\hline 21 & 0.012 & -0.007 & -0.012 & 0.001 & 0.001 & -0.012 & 0.013 \\
\hline 22 & -0.013 & 0.012 & 0.008 & -0.001 & -0.001 & 0.009 & -0.013 \\
\hline 23 & 0.007 & -0.047 & -0.006 & 0.001 & 0.001 & -0.006 & 0.013 \\
\hline 24 & -0.010 & 0.068 & 0.007 & -0.001 & -0.001 & 0.006 & -0.007 \\
\hline 25 & & -0.077 & -0.006 & 0.001 & 0.000 & -0.010 & 0.057 \\
\hline 26 & & 0.963 & 0.048 & 0.000 & -0.001 & 0.021 & -0.069 \\
\hline 27 & & & & & 0.000 & 0.028 & -0.045 \\
\hline 28 & & & & & 0.001 & -0.012 & 0.065 \\
\hline 29 & & & & & 0.000 & 0.031 & -0.072 \\
\hline 30 & & & & & 0.001 & -0.023 & 0.947 \\
\hline
\end{tabular}

${ }^{a}$ The numbering of carbon atoms corresponds to that given in Figure S2. 
TABLE S2: Absolute Vibrationless Energies (hartrees), Unscaled Zero-point Vibrational Energies (ZPVE, hartrees) and Unscaled Thermal Corrections to the Enthalpy $(\Delta \Delta H, \text { hartrees })^{a}$

\begin{tabular}{|c|c|c|c|c|c|c|c|}
\hline PAH & Energy & ZPVE & $\Delta \Delta H$ & PAH & Energy & ZPVE & $\Delta \Delta H$ \\
\hline B & -232.15166 & 0.10016 & 0.10550 & BA & -692.90238 & 0.24009 & 0.25318 \\
\hline $\mathbf{N}$ & -385.73550 & 0.14693 & 0.15470 & DBA & -846.49046 & 0.28675 & 0.30260 \\
\hline A & -539.31255 & 0.19333 & 0.20374 & BNA & -1000.06473 & 0.33361 & 0.35203 \\
\hline $\mathbf{T}$ & -692.88674 & 0.23959 & 0.25270 & BPHA & -1153.64156 & 0.37986 & 0.40099 \\
\hline $\mathbf{P}$ & -846.45941 & 0.28578 & 0.30162 & $\mathbf{C}$ & -921.54052 & 0.27865 & 0.29372 \\
\hline PH & -539.32239 & 0.19366 & 0.20408 & PYR & -615.52867 & 0.20641 & 0.21741 \\
\hline BPH & -692.89692 & 0.24055 & 0.25352 & PER & -769.10206 & 0.25294 & 0.26687 \\
\hline DBPH & -846.47365 & 0.28680 & 0.30244 & BPER & -845.32391 & 0.26571 & 0.28021 \\
\hline TS & E & ZPVE & $\Delta \Delta H$ & $\begin{array}{l}\text { Aryl } \\
\text { radical }\end{array}$ & EI & ZPVE & $\Delta \Delta H$ \\
\hline TS:B-1 & -271.93743 & 0.12800 & 0.13655 & B-1 & -231.46387 & 0.08703 & 0.09235 \\
\hline TS:N-1 & -425.52106 & 0.17490 & 0.18583 & $\mathbf{N}-1$ & -385.04734 & 0.13397 & 0.14173 \\
\hline TS:N-2 & -425.52121 & 0.17469 & 0.18572 & $\mathbf{N}-2$ & -385.04763 & 0.13379 & 0.14155 \\
\hline TS:A-1 & -579.09748 & 0.22147 & 0.23510 & A-1 & -538.62383 & 0.18064 & 0.19104 \\
\hline TS:A-2 & -579.09813 & 0.22128 & 0.23487 & A-2 & -538.62441 & 0.18034 & 0.19074 \\
\hline TS:A-3 & -579.09843 & 0.22108 & 0.23476 & A-3 & -538.62462 & 0.18010 & 0.19052 \\
\hline TS:T-1 & -732.67173 & 0.26771 & 0.28404 & $\mathbf{T}-1$ & -692.19797 & 0.22685 & 0.23996 \\
\hline TS:T-2 & -732.67242 & 0.26744 & 0.28377 & T-2 & -692.19869 & 0.22655 & 0.23967 \\
\hline
\end{tabular}


TABLE S2: continued ...

$\begin{array}{llllllll}\text { TS:T-3 } & -732.67271 & 0.26731 & 0.28372 & \text { T-3 } & -692.19899 & 0.22639 & 0.23951 \\ \text { TS:P-1 } & -886.24429 & 0.31371 & 0.33279 & \text { P-1 } & -845.77048 & 0.27276 & 0.28860 \\ \text { TS:P-2 } & -886.24444 & 0.31378 & 0.33283 & \text { P-2 } & -845.77061 & 0.27283 & 0.28868 \\ \text { TS:P-3 } & -886.24525 & 0.31358 & 0.33268 & \text { P-3 } & -845.77135 & 0.27267 & 0.28852 \\ \text { TS:P-4 } & -886.24542 & 0.31347 & 0.33261 & \text { P-4 } & -845.77169 & 0.27237 & 0.28826 \\ \text { TS:PH-1 } & -579.10694 & 0.22207 & 0.23546 & \text { PH-1 } & -538.63692 & 0.18062 & 0.19100 \\ \text { TS:PH-2 } & -579.10822 & 0.22146 & 0.23516 & \text { PH-2 } & -538.63446 & 0.18057 & 0.19097 \\ \text { TS:PH-3 } & -579.10805 & 0.22146 & 0.23515 & \text { PH-3 } & -538.63406 & 0.18058 & 0.19099 \\ \text { TS:PH-4 } & -579.10817 & 0.22176 & 0.23533 & \text { PH-4 } & -538.63425 & 0.18076 & 0.19116 \\ \text { TS:PH-5 } & -579.10819 & 0.22167 & 0.23527 & \text { PH-5 } & -538.63438 & 0.18070 & 0.19112 \\ \text { TS:BPH-1 } & -732.68167 & 0.26868 & 0.28461 & \text { BPH-1 } & -692.21855 & 0.22687 & 0.23998 \\ \text { TS:BPH-2 } & -732.68272 & 0.26833 & 0.28455 & \text { BPH-2 } & -692.20927 & 0.22741 & 0.24036 \\ \text { TS:BPH-3 } & -732.68248 & 0.26835 & 0.28460 & \text { BPH-3 } & -692.20858 & 0.22743 & 0.24038 \\ \text { TS:BPH-4 } & -732.68253 & 0.26860 & 0.28472 & \text { BPH-4 } & -692.20894 & 0.22761 & 0.24056 \\ \text { TS:BPH-5 } & -732.68233 & 0.26853 & 0.28470 & \text { BPH-5 } & -692.20852 & 0.22760 & 0.24056 \\ \text { TS:BPH-6 } & -732.68253 & 0.26852 & 0.28470 & \text { BPH-6 } & -692.20878 & 0.22759 & 0.24056 \\ \text { TS:DBPH-5 } & -886.25888 & 0.31482 & 0.33367 & \text { DBPH-5 } & -845.78513 & 0.27384 & 0.28948 \\ \text { TS:DBPH-1 } & -886.25883 & 0.31492 & 0.33355 & \text { DBPH-1 } & -845.79016 & 0.27362 & 0.28929 \\ \text { TS:DBPH-2 } & -886.25931 & 0.31457 & 0.33349 & \text { DBPH-2 } & -845.78570 & 0.27365 & 0.28928 \\ \text { TS:DBPH-3 } & -886.25887 & 0.31456 & 0.33349 & \text { DBPH-3 } & -845.78536 & 0.27371 & 0.28933 \\ & -886.25934 & 0.31484 & 0.33367 & \text { DBPH-6 } & -845.78562 & 0.27385 & 0.28949\end{array}$


TABLE S2: continued ...

\begin{tabular}{|c|c|c|c|c|c|c|c|}
\hline TS:DBPH-7 & -886.25881 & 0.31481 & 0.33366 & DBPH-7 & -845.78512 & 0.27388 & 0.28951 \\
\hline TS:BA-1 & -732.68671 & 0.26844 & 0.28454 & BA-1 & -692.21696 & 0.22698 & 0.24006 \\
\hline TS:BA-2 & -732.68481 & 0.26882 & 0.28475 & $\mathbf{B A}-2$ & -692.21634 & 0.22724 & 0.24032 \\
\hline TS:BA-3 & -732.68786 & 0.26803 & 0.28433 & BA-3 & -692.21413 & 0.22706 & 0.24017 \\
\hline TS:DBA-1 & -886.27093 & 0.31593 & 0.33436 & DBA-1 & -845.80726 & 0.27386 & 0.28964 \\
\hline TS:DBA-2 & -886.27523 & 0.31527 & 0.33405 & DBA-2 & -845.80542 & 0.27374 & 0.28953 \\
\hline TS:DBA-3 & -886.27632 & 0.31449 & 0.33366 & DBA-3 & -845.80249 & 0.27366 & 0.28950 \\
\hline TS:BNA-1 & -1039.85047 & 0.36179 & 0.38317 & BNA-1 & -999.38668 & 0.31992 & 0.3384 \\
\hline TS:BNA-2 & -1039.84787 & 0.36224 & 0.38341 & BNA-2 & -999.38810 & 0.32001 & 0.3376 \\
\hline TS:BNA-3 & -1039.84922 & 0.36198 & 0.38338 & BNA-3 & -999.37931 & 0.32052 & 0.33 \\
\hline TS:BPHA-1 & -1193.42572 & 0.40830 & 0.43234 & ВРНА-1 & -1152.95553 & 0.36676 & 0.3878 \\
\hline TS:BPHA-2 & -1193.42429 & 0.40864 & 0.43247 & ВРНА-2 & -1152.96098 & 0.36690 & 0.38800 \\
\hline TS:BPHA-3 & -1193.42694 & 0.40795 & 0.43208 & ВРНА-3 & -1152.95904 & 0.36674 & 0.38788 \\
\hline TS:C-1 & -961.32585 & 0.30657 & 0.32489 & C-1 & -920.85160 & 0.26564 & 0.2807 \\
\hline TS:PYR-1 & -655.31398 & 0.23438 & 0.24862 & PYR-1 & -614.84005 & 0.19366 & 0.2046 \\
\hline TS:PYR-2 & -655.31445 & 0.23415 & 0.24847 & PYR-2 & -614.84084 & 0.19328 & 0.2042 \\
\hline TS:PYR-3 & -655.31459 & 0.23443 & 0.24863 & PYR-3 & -614.84068 & 0.19349 & 0.2045 \\
\hline TS:PER-1 & -808.88640 & 0.28132 & 0.29816 & PER-1 & -768.41690 & 0.25369 & 0.2536 \\
\hline $\mathrm{P}$ & 5.10836 & 0.29415 & 0.31165 & BPEI & -844.63807 & 0.26722 & 0.2 \\
\hline
\end{tabular}

${ }^{a}$ Energies calculated at the BMK/6-311+G(3df,2p)//B3-LYP/6-311G(d,p) level of theory, ZPVE and $\Delta \Delta H$ calculated at the level of geometry optimization, i.e. B3-LYP/6-311G(d,p). 
TABLE S3: Effect of Internal Rotation and Eckart Tunneling Corrections on Activation Energies $E_{\mathrm{a}}\left(\mathrm{kJ} \mathrm{mol}^{-1}\right)$ and Pre-Exponential Factors $A\left(\mathrm{~m}^{3} \mathrm{~mol}^{-1} \mathrm{~s}^{-1}\right)$ for the Abstraction Reactions in the Temperature Interval 700-1100 K

\begin{tabular}{|c|c|c|c|c|c|c|}
\hline & \multicolumn{3}{|c|}{$E_{\mathrm{a}}$} & \multicolumn{3}{|c|}{$A$} \\
\hline & $\mathrm{HO}$ & $\begin{array}{c}\mathrm{HO} / \mathrm{FR} \text { or } \\
\mathrm{HO} / \mathrm{HR}\end{array}$ & $\begin{array}{l}\text { Mixed + } \\
\text { tunneling }\end{array}$ & $\mathrm{HO}$ & $\begin{array}{c}\mathrm{HO} / \mathrm{FR} \text { or } \\
\mathrm{HO} / \mathrm{HR}\end{array}$ & $\begin{array}{l}\text { Mixed + } \\
\text { tunneling }\end{array}$ \\
\hline B-1 & 88.79 & 85.16 & 80.90 & $1.29 \times 10^{9}$ & $3.44 \times 10^{7}$ & $2.62 \times 10^{7}$ \\
\hline $\mathbf{N}-1$ & 89.57 & 85.93 & 81.63 & $6.62 \times 10^{7}$ & $2.23 \times 10^{7}$ & $1.69 \times 10^{7}$ \\
\hline $\mathbf{N}-2$ & 88.99 & 85.36 & 81.13 & $3.18 \times 10^{8}$ & $2.44 \times 10^{7}$ & $1.86 \times 10^{7}$ \\
\hline A-1 & 91.67 & 88.04 & 83.57 & $1.74 \times 10^{8}$ & $1.60 \times 10^{7}$ & $1.20 \times 10^{7}$ \\
\hline $\mathbf{A}-2$ & 89.52 & 85.89 & 81.58 & $6.32 \times 10^{7}$ & $2.04 \times 10^{7}$ & $1.55 \times 10^{7}$ \\
\hline A-3 & 88.53 & 84.90 & 80.66 & $1.72 \times 10^{8}$ & $2.57 \times 10^{7}$ & $1.96 \times 10^{7}$ \\
\hline $\mathbf{T}-1$ & 91.48 & 87.85 & 83.38 & $8.77 \times 10^{7}$ & $1.60 \times 10^{7}$ & $1.20 \times 10^{7}$ \\
\hline $\mathbf{T}-2$ & 89.17 & 85.53 & 81.23 & $7.12 \times 10^{7}$ & $2.05 \times 10^{7}$ & $1.55 \times 10^{7}$ \\
\hline $\mathbf{T}-3$ & 88.28 & 84.65 & 80.43 & $2.00 \times 10^{8}$ & $2.68 \times 10^{7}$ & $2.04 \times 10^{7}$ \\
\hline $\mathbf{P}-1$ & 91.49 & 87.86 & 83.37 & $1.33 \times 10^{8}$ & $1.62 \times 10^{7}$ & $1.21 \times 10^{7}$ \\
\hline $\mathbf{P}-2$ & 91.15 & 87.52 & 83.04 & $7.47 \times 10^{7}$ & $1.59 \times 10^{7}$ & $1.19 \times 10^{7}$ \\
\hline $\mathbf{P}-3$ & 88.69 & 85.06 & 80.75 & $1.08 \times 10^{8}$ & $2.26 \times 10^{7}$ & $1.71 \times 10^{7}$ \\
\hline P-4 & 88.13 & 84.50 & 80.26 & $1.97 \times 10^{8}$ & $2.64 \times 10^{7}$ & $2.02 \times 10^{7}$ \\
\hline PH-1 & 92.50 & 89.70 & 84.82 & $1.37 \times 10^{7}$ & $8.07 \times 10^{6}$ & $5.85 \times 10^{6}$ \\
\hline PH-2 & 88.71 & 85.08 & 80.85 & $1.93 \times 10^{8}$ & $1.15 \times 10^{7}$ & $8.78 \times 10^{6}$ \\
\hline PH-3 & 89.11 & 85.48 & 81.29 & $8.80 \times 10^{7}$ & $1.21 \times 10^{7}$ & $9.27 \times 10^{6}$ \\
\hline PH-4 & 89.13 & 85.49 & 81.18 & $2.66 \times 10^{7}$ & $9.09 \times 10^{6}$ & $6.89 \times 10^{6}$ \\
\hline
\end{tabular}


TABLE S3: continued ....

\begin{tabular}{|c|c|c|c|c|c|c|}
\hline PH-5 & 89.02 & 85.38 & 81.07 & $2.91 \times 10^{7}$ & $1.10 \times 10^{7}$ & $8.32 \times 10^{6}$ \\
\hline ВРН-1 & 91.47 & 88.40 & 83.33 & $3.87 \times 10^{6}$ & $2.41 \times 10^{6}$ & $1.71 \times 10^{6}$ \\
\hline ВРН-2 & 88.75 & 85.11 & 80.88 & $2.78 \times 10^{7}$ & $6.22 \times 10^{6}$ & $4.74 \times 10^{6}$ \\
\hline ВРН-3 & 89.45 & 85.82 & 81.63 & $4.67 \times 10^{7}$ & $6.40 \times 10^{6}$ & $4.90 \times 10^{6}$ \\
\hline ВРН-4 & 89.58 & 85.95 & 81.62 & $1.22 \times 10^{7}$ & $5.07 \times 10^{6}$ & $3.84 \times 10^{6}$ \\
\hline ВРН-5 & 90.07 & 86.44 & 82.16 & $1.65 \times 10^{7}$ & $5.52 \times 10^{6}$ & $4.20 \times 10^{6}$ \\
\hline ВРН-6 & 89.56 & 85.92 & 81.62 & $1.73 \times 10^{7}$ & $5.77 \times 10^{6}$ & $4.38 \times 10^{6}$ \\
\hline DBPH-1 & 90.39 & 87.61 & 82.80 & $3.63 \times 10^{6}$ & $1.95 \times 10^{6}$ & $1.41 \times 10^{6}$ \\
\hline DBPH-2 & 89.12 & 85.49 & 81.28 & $3.99 \times 10^{7}$ & $6.18 \times 10^{6}$ & $4.72 \times 10^{6}$ \\
\hline DВРН-3 & 90.25 & 86.62 & 82.42 & $7.55 \times 10^{7}$ & $6.07 \times 10^{6}$ & $4.64 \times 10^{6}$ \\
\hline DBPH-4 & 90.09 & 86.46 & 82.13 & $1.26 \times 10^{7}$ & $4.67 \times 10^{6}$ & $3.54 \times 10^{6}$ \\
\hline DBPH-5 & 90.58 & 86.94 & 82.68 & $1.62 \times 10^{7}$ & $5.10 \times 10^{6}$ & $3.88 \times 10^{6}$ \\
\hline DВРН-6 & 89.38 & 85.74 & 81.45 & $1.34 \times 10^{7}$ & $5.02 \times 10^{6}$ & $3.81 \times 10^{6}$ \\
\hline DВРН-7 & 90.77 & 87.13 & 82.87 & $1.61 \times 10^{7}$ & $5.17 \times 10^{6}$ & $3.93 \times 10^{6}$ \\
\hline BA-1 & 93.10 & 90.49 & 85.62 & $9.28 \times 10^{6}$ & $6.12 \times 10^{6}$ & $4.44 \times 10^{6}$ \\
\hline BA-2 & 98.39 & 96.67 & 91.61 & $2.13 \times 10^{6}$ & $1.36 \times 10^{6}$ & $9.71 \times 10^{5}$ \\
\hline BA-3 & 89.83 & 86.19 & 81.87 & $1.76 \times 10^{7}$ & $5.91 \times 10^{6}$ & $4.48 \times 10^{6}$ \\
\hline DBA-1 & 103.64 & 102.09 & 96.67 & $1.18 \times 10^{6}$ & $1.07 \times 10^{6}$ & $7.44 \times 10^{5}$ \\
\hline DBA-2 & 92.05 & 89.25 & 84.36 & $1.23 \times 10^{7}$ & $9.72 \times 10^{6}$ & $7.03 \times 10^{6}$ \\
\hline DBA-3 & 88.61 & 84.98 & 80.75 & $3.33 \times 10^{7}$ & $1.25 \times 10^{7}$ & $9.54 \times 10^{6}$ \\
\hline BNA-1 & 88.92 & 85.95 & 80.93 & $3.07 \times 10^{6}$ & $1.72 \times 10^{6}$ & $1.23 \times 10^{6}$ \\
\hline BNA-2 & 96.04 & 93.95 & 88.45 & $1.02 \times 10^{6}$ & $7.72 \times 10^{5}$ & $5.30 \times 10^{5}$ \\
\hline
\end{tabular}


TABLE S3: continued ...

$\begin{array}{lcccccc}\text { BNA-3 } & 92.60 & 89.73 & 84.92 & 3.50 \times 10^{6} & 1.53 \times 10^{6} & 1.11 \times 10^{6} \\ \text { BPHA-1 } & 93.47 & 90.77 & 86.00 & 2.56 \times 10^{6} & 1.55 \times 10^{6} & 1.13 \times 10^{6} \\ \text { BPHA-2 } & 97.33 & 95.38 & 89.99 & 8.62 \times 10^{5} & 7.69 \times 10^{5} & 5.33 \times 10^{5} \\ \text { BPHA-3 } & 89.87 & 86.96 & 82.14 & 3.98 \times 10^{6} & 2.27 \times 10^{6} & 1.64 \times 10^{6} \\ \text { C-1 } & 90.25 & 86.62 & 82.34 & 2.39 \times 10^{8} & 6.28 \times 10^{7} & 4.77 \times 10^{7} \\ \text { PYR-1 } & 90.35 & 86.71 & 82.46 & 9.06 \times 10^{7} & 2.21 \times 10^{7} & 1.68 \times 10^{7} \\ \text { PYR-2 } & 88.85 & 85.23 & 80.99 & 3.80 \times 10^{8} & 2.52 \times 10^{7} & 1.92 \times 10^{7} \\ \text { PYR-3 } & 88.77 & 85.14 & 80.83 & 6.13 \times 10^{7} & 2.25 \times 10^{7} & 1.71 \times 10^{7} \\ \text { PER-1 } & 92.84 & 90.18 & 85.35 & 1.10 \times 10^{7} & 6.79 \times 10^{6} & 4.93 \times 10^{6} \\ \text { BPER-1 } & 92.87 & 90.09 & 85.21 & 2.24 \times 10^{7} & 1.40 \times 10^{7} & 1.01 \times 10^{7}\end{array}$

